\title{
Patient perceptions of students in a longitudinal integrated clerkship in Taiwan: a qualitative study
}

\author{
Yaw-Wen Chang ${ }^{1,2}$ (D) David A. Hirsh ${ }^{3,4} \mathbb{B}$, Wen-Hui Fang ${ }^{1} \mathbb{B}$, Honghe Li ${ }^{5} \mathbb{D}$, Wen-Chii Tzeng ${ }^{6} \mathbb{D}$ and
} Senyeong $\mathrm{KaO}^{7 *}$ (D)

\begin{abstract}
Background: Longitudinal integrated clerkships (LICS) are a model of clinical education growing rapidly in Western contexts. LICs use educational continuity to benefits students' clinical learning and professional identity formation. Patient-centered care is a core component of medical professionalism in the West. To support patient-centered care, education leaders in Taiwan restructured clinical education and implemented the first longitudinal integrated clerkship in East Asia. We aimed to investigate patients' perceptions of longitudinal relationships with the LIC students within Taiwan's Confucian cultural and social context.

Methods: We invited patients or their family members who were cared for longitudinally by a LIC student to participate in the study. Participating patients or their family members undertook semi-structured interviews. We analyzed data qualitatively using a general inductive approach to identify themes in the patients' descriptions of their experiences interacting with the LIC students.

Results: Twenty-five patients and family members participated in interviews: 16 patients and 9 family members. Qualitative analysis of interview transcripts identified three themes from patients' experience receiving care from their LIC students: care facilitation, companionship, and empathy. To provide care facilitation, LIC students served as a bridge between the physicians and patients. Students served patients by reminding, consulting, tracking disease progression, and researching solutions for problems. To provide companionship, students accompanied patients interpersonally like a friend or confidant who listens and provides a presence for patients. To provide empathy, patients reported that students showed sincere concern for patients' experience, feelings, and mood.

(Continued on next page)
\end{abstract}

\footnotetext{
* Correspondence: kao@mail.ndmctsgh.edu.tw

${ }^{7}$ Graduate Institute of Life Science, National Defense Medical Center, No.161,

Sec. 6, Minquan E. Rd., Neihu Dist., Taipei City 11490, Taiwan, Republic of

China

Full list of author information is available at the end of the article
}

(C) The Author(s). 2021 Open Access This article is licensed under a Creative Commons Attribution 4.0 International License, which permits use, sharing, adaptation, distribution and reproduction in any medium or format, as long as you give appropriate credit to the original author(s) and the source, provide a link to the Creative Commons licence, and indicate if changes were made. The images or other third party material in this article are included in the article's Creative Commons licence, unless indicated otherwise in a credit line to the material. If material is not included in the article's Creative Commons licence and your intended use is not permitted by statutory regulation or exceeds the permitted use, you will need to obtain permission directly from the copyright holder. To view a copy of this licence, visit http://creativecommons.org/licenses/by/4.0/ The Creative Commons Public Domain Dedication waiver (http://creativecommons.org/publicdomain/zero/1.0/) applies to the data made available in this article, unless otherwise stated in a credit line to the data. 
(Continued from previous page)

Conclusion: In our study, Taiwanese patients' perspectives of LIC students suggested the value of care facilitation, companionship, and empathy. We discuss these themes within the context of Confucian culture and the Taiwanese context of care.

Keywords: Undergraduate medical education, Medical student, Longitudinal integrated clerkship, Relationship, Continuity, Patient centered, Patient perception, Professionalism, Empathy, East Asia, Qualitative study

\section{Background}

Education leaders create longitudinal integrated clerkships (LICs) to advance students' clinical and scientific learning and support their professional identity formation [1-5]. In contrast to traditional block rotations, LICs restructure clinical education to provide students' educational continuity-continuity of care, curriculum, and supervision [5-8]. Through continuity, LICs reshape learning relationships and the learning environment $[9,10]$. The literature on LICs reports educational benefits $[4,5,11]$, and LICs support humanistic professional identity formation [12, 13], increase patient-centeredness $[1,3,11,14]$, and may improve communication skills $[5,15,16]$. Nonetheless, LIC research has been conducted primarily in the US, Canada, Europe, and Australia [17, 18].

The literature describes Western and East Asian conceptualizations of medical professionalism [19-22]. In these contexts, patients' experience of care relates to patients' self-concepts and patterns of self-other relationships, that are primarily culturally determined [23]. Authors describe differences between cultures even as commonalities exist across Western and East Asian contexts, heterogeneity exists within contexts, and both Western and East Asian conceptualizations are dynamic and changing [24]. In Taiwan and Mainland China, the medical professionalism framework has developed on the basis of Confucianism. This framework emphasizes personal integrity, benevolence, and collective social relations [19, 25-27]. The literature suggests that, consistent with Confucian tradition, East Asian patients expect the physician-patient relationship and the physician's role to involve greater physician paternalism and authority [24]. Compared with patients in the West, East Asian patients may be less inclined to question or express concerns to their physician [24]. Western contexts appear to more strenuously evince the values of patient autonomy and physician-patient "shared-decision making" [21, 24]. In addition, in East Asian contexts influenced by Confucianism, patients--and physicians--also expect the close involvement of, even deference to, the patient's family [21, 24]. In East Asia, cultural shifts and policies now promote patient-centeredness, although change has been slow [24]. In this Confucian culture context, it remains unclear the degree to which cultural or institutional influences support or impede these changes [24]. The literature also reports social contextual factors, including the health care system, government investment in healthcare, health insurance, and patient health literacy, impact patient experience of healthcare $[28,29]$.

To support patient-centered care, education leaders in Taipei, Taiwan restructured clinical education and implemented a LIC in a tertiary teaching hospital. The program intended that longitudinal relationships in LICs would support patient-centeredness. We developed our study's conceptual framework by considering the principle of educational continuity and the longitudinal relationships that underpin the LIC structure [6,30]. We theorize that just as students experience patientcentered beliefs arising from longitudinal relationships [31], patients are also affected by their longitudinal connection to students [32,33]. Although the LIC literature does report patients' positive reports of having LIC students in Western countries [32-34], we are aware of no studies in the Asian context of patients' experiences of LICs. In this study, we investigated patients' perceptions of longitudinal relationships with the LIC students in the Taiwan's Confucian cultural and social context.

\section{Methods}

\section{Introduction of the Tri-Service General Hospital} longitudinal integrated clerkship

In Taiwan, medical students undergo 6-year medical programs beginning after high school. In this study, the LIC students came from the medical school of the National Defense Medical Center (NDMC) located in Taipei, a city of 2.7 million inhabitants [35] At NDMC, medical students enter clinical clerkships in their 5th year, followed by sub-internships in the 6th year. The core clerkships begin in October and end in May, and include internal medicine, surgery, gynecology/obstetrics, pediatrics, psychiatry, family medicine, diagnostic radiology, and clinical pathology. These rotations take place in the Tri-Service General Hospital (TSGH), a tertiary teaching hospital located in Taipei City. The Tri-Service General Hospital longitudinal integrated clerkship (TSGH LIC) provides two clerkship structures: a traditional block rotation and an LIC. The LIC takes place in the first 6 months of the core clerkship (5th) year. All clerkships at TSGH are hospital-based, because 
that is the principal model care delivery at NDMC. The LIC is a blended-type LIC [18] that occurs predominantly in TSGH's tertiary inpatient setting. Medical students join this program voluntarily and are selected by lottery. Approximately 12 students enter this program each year (about $10 \%$ of the class).

The program pairs every LIC student with preceptors from each of the following disciplines: internal medicine, surgery, obstetrics/gynecology, pediatrics, psychiatry, and family medicine. The first 8 weeks of the LIC program are the inpatient immersion stage, during which the students are required to rotate on the wards of internal medicine, surgery, pediatrics, and gynecology/obstetric every 2 weeks. In the four months following the immersion, the students begin the integrated clinical learning and learning with their preceptors of six disciplines simultaneously. In this stage, the students primarily work with inpatient care teams and follow their patients into outpatient clinics. Preceptors assign longitudinal care patients to the LIC students based on the learning objectives and the patients' conditions. The students will follow their patients by telephone or communication apps while patients are not hospitalized. They complete the follow-up through the course of patients' treatment or until the conclusion of the LIC program.

\section{Study design and sample}

We performed a qualitative study based on semistructured interviews with patients or their family members who were being cared for and tracked longitudinally by LIC students in the academic year 2016-17. Interviews occurred between March to June 2017. We reviewed students' patient logs, and invited all patients or their family members who fulfilled the following criteria to participate in this study: (1) the patient was cared for by a LIC student during the patient's hospitalization; and (2) the patient had 3 or more contacts with a LIC student during an outpatient clinic, emergency visit, or rehabilitation after the hospitalization. If a patient was under 18 years old or had problems with verbal communication, consent was provided by the patient's legal guardian, and this caregiver or parent was interviewed instead of, or along with, the patient.

The institutional review board of TSGH approved this study (TSGHIRB 2-105-05-050).

\section{Data collection}

A trained research assistant, with no connection with the LIC program, conducted face-to-face interviews with LIC students' patients or their family "guardian" using a predetermined semi-structured interview guide (Additional file 1). The research assistant asked the interviewees to describe how medical students were involved in the care process and how the patients felt about the involvement of the medical students. The research assistant transcribed the audio recordings verbatim and de-identified the transcripts. The primary investigators cross-checked the verbatim transcripts with the content of the original sound recordings to ensure the accuracy of the transcripts.

\section{Data analysis}

We employed a general inductive approach to analyze the verbatim transcripts $[36,37]$. We sought to identify themes that might emerge from patients' experience of receiving care from their LIC students. Two primary investigators ( $\mathrm{YC}$, director of LIC program and WF, a preceptor the LIC program) first read 3 to 5 interview transcripts and separately performed the initial coding. Subsequently, they compared their work, discussed each other's codes, and formed the initial codebook. In the next step, the two primary investigators and a research assistant coded another 3 to 5 interview transcripts according to these codes. Using the same process of deliberation and integration, these three researchers examined and discussed the codes, and refined the codebook. Hereafter, the primary investigators (YC, WF) and the research assistant coded the remaining interview transcripts using the coding instruction manual; the research assistant read and coded all the interview transcripts, whereas the two primary investigators each read and coded half of the interview transcripts. Thereby, each interview transcript was co-completed by the research assistant and one of the primary investigators. After completing the initial coding process, the two primary investigators ( $\mathrm{YC}$, WF) iteratively inspected and discussed all codes and categories, and generated the themes. In the final step, the primary investigators explained the themes and examples to all members of the research team (DAH, HL, WT, SK); the team then examined and discussed these results together to establish consensus.

\section{Results}

The LIC students' patient logs listed 57 longitudinal care patients who met the study's two inclusion criteria. All patients were invited to be interviewed. The final study sample consisted of 20 patients. These patients comprised 25 interviewees: 16 patient interviewees and 9 family members (Table 1). Respondents under 18 years old were interviewed with or substituted by at least one parent or guardian. Patients in the final sample included recruits from all 12 students. All interviews were conducted face-to-face. The average length of interviews was $50 \mathrm{~min}$ (range $43 \mathrm{~min}$ to $68 \mathrm{~min}$ ). Twenty-two 
Table 1 Identity and sex of the interviewees

\begin{tabular}{lll}
\hline \multicolumn{2}{c}{$\mathbf{n}(\%)$} & Interviewee label \\
\hline Interviewee identity & & \\
Patient & $16(64 \%)$ & P002, P003, P004-1, P005, P006, P007, P008, P009-1, P011-1, P012-1, P013, P014, P015, P017, P018, P019 \\
Family member & $9(36 \%)$ & P001-1, P001-2, P004-2, P009-2, P010, P011-2, P012-2, P016, P020 \\
Sex & \\
Male & $7(28 \%)$ & \\
Female & $18(72 \%)$ & \\
Age & $41.6 \pm 15.7$ years old \\
\hline
\end{tabular}

patients turned down the interview invitation; 12 could not be reached by telephone, and 3 passed away. Of the 25 interviewees, 18 (72\%) were female, and 7 (28\%) were male. There was no significant difference in the proportion of females between participants (patients or their participating family members) and patients not interviewed ( $P>.05$ for each comparison). The interviewees had an average age of 41.6 years; the youngest was 13 years old and the eldest 73 years old. There was no significant difference in the distribution of age between participants (patients or their participating family members) and patients not interviewed ( $P>.05$ for each comparison). Other than sex and age, we did not collect information from patients who declined to participate.
We identified 3 themes from the patient interviews: care facilitation, companionship, and empathy (Fig. 1); we present themes and representative quotations for each theme. We summarize the findings below:

\section{Care facilitation}

The interviewees recognize the student as a resource, connecting patients to their attending and improving patients' access to the healthcare system. They mentioned their interactions with LIC students during hospitalization periods or at outpatient clinic sessions. The medical students kept in contact with the patients with telephone or instant messaging app. They reminded and confirmed the appointment date with patients, tracking their response to treatment, provided health education, and facilitated their care.

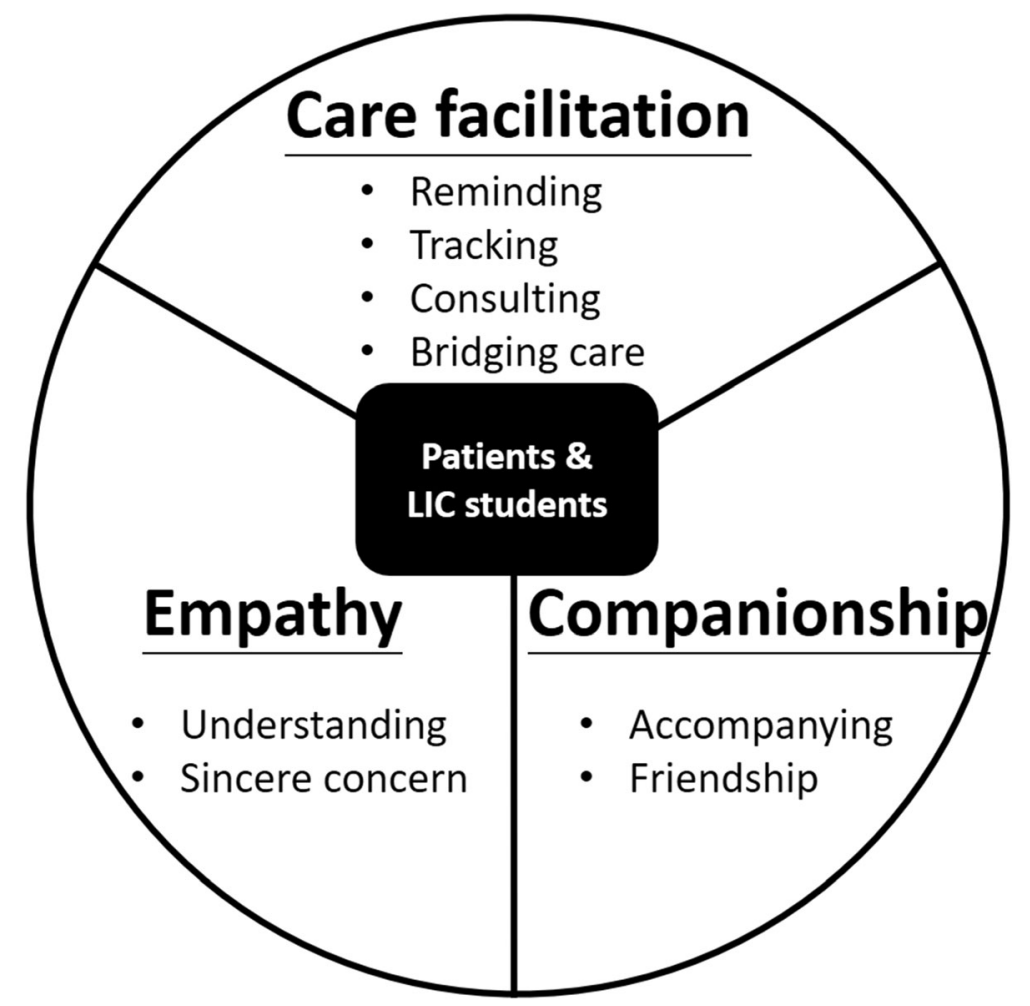

Fig. 1 Major themes from patients' descriptions of students' participation in their longitudinal clinical care 
"The medical student will LINE (a chat app) me and confirm the date of visit." (P018)

"We chatted on the LINE. The students taught me how to control my diet in order to lower my blood sugar." (P014)

The interviewees described their student as a sort of consultant alongside the attending physician. The student not only provided consulting information but also offered them additional information about the hospital.

"I will ask him (the medical student) which pediatrician is better at allergy or what. He is quite clear. He knows what the expertise of each doctor is." (P002)

"In addition to the attending physician, you have the option of consulting other people, so you get the feeling of having a consultant team." (P003).

They expressed that LIC students strengthened the bridge between the attending physician and the patient. When patients would raise questions, the students provided answers directly or facilitated connection to the attending physician.

"It is not easy to find my doctor. I can ask them [students] to help me ask. It may take some time, but I always get solutions to my problems." (P005)

"They pass the message on to my attending physician. The physician then examines the situation and gives instructions." (P001-1)

\section{Companionship}

The interviewees perceive the students' presence. The students visited them daily while they stayed in the hospital, met them while they came back to the outpatient clinic, and kept in contact with them by telephone or communication apps when they were home. Numerous interviewees revealed that the LIC students were like a companion with professional knowledge and skills. They noted that students spent considerable time accompanying patients.

"If I come to the hospital or clinic, he [the medical student] will come to see me, as long as I come to the hospital, he will look after me." (P015)

"When we have a problem, we first think of him (medical student). Because we can only see the doctor at a certain time, but we can contact him (medical student) anytime." (P011-1)
Interviewees shared that the students cared about their wellbeing and shared relevant knowledge with them. With the students' company and assistance, they would be less worried and feel at ease. Sometimes, the patients described students like friends they can talk to.

"I feel like [the medical student] is a good friend, a very good friend. She is very caring and gave me a caring feeling." (P001-2)

"Sometimes, there are things you don't want to talk to your family, [... ] don't want to talk to the doctor. Medical students are more like friends, and sometimes you want to talk to friends about something." (P012-1)

Some interviewees expressed that they expected to see the students when they went back to outpatient visits, and like meeting a friend, the connection offered benefit.

"It likes that when my son was going to see a doctor, he seemed going to have an appointment with his friends. He will feel a little happier because friends will come to see him in the waiting room." (P009-2)

\section{Empathy}

The interviewees perceive the students' attend to the patients' feelings. Some interviewees explained that through longitudinal care, the LIC students could more deeply consider the patients' experiences of being sick and patients' experience of the treatment process. They expressed that they valued students' displays of empathy.

"They probably have a better understanding of the stress of having a chronic disease after they have gained experience of such contact with us [patients or family members]. They will be more empathetic." (P011-2)

"They have a sense of empathy; they know the physical and emotional discomfort we are facing, so they comfort us in their own ways and help us snap out of our bad moods." (P006)

The interviewees also shared that students could better learn the families' experiences about caring for these patients. They noted that medical students could understand their distress and support them in hard times.

"She [the medical student] is not only focusing on my son [the patient] but also concerning my feelings. I feel being looking after." (P020) 
"In the process of communicating with medical students, you gradually know their professional abilities, and then they also understand your suffering and your blind spots." (P010)

The interviewees expressed that they received sincere concern from the students that were beyond the standard clinical diagnosis and treatment process.

"They don't make me think that I am a patient. They can chat with me and make me feel like a friend. Because they may be about the same age, they can understand my thoughts well". (P014)

"I was discharged from the hospital, but my baby has not been discharged yet. The medical student promised me to see my baby every day and report to me my baby's condition." (P008)

\section{Discussion}

In this qualitative study, we used an inductive approach to analyze interviews of LIC students' longitudinal patients or their family members. We extracted three themes: care facilitation, companionship, and empathy. To provide care facilitation, LIC students serve as a bridge between the physicians and patients. Students serve patients by reminding, consulting, tracking disease progression, and researching solutions for problems. When providing companionship, students accompany patients interpersonally like a friend or confidant who listens and provides a presence for patients. To provide empathy, patients reported that students showed sincere concern for patients' experience, feelings, and mood. To our knowledge, this is the first study characterizing patients' perspectives of their engagement with longitudinal students in the Asian context.

Our study connects to prior studies in the Western context that examined LIC students' experiences with patients. In our context, the patients recognized their students as a resource to facilitate care. Patients described the benefits when students connected them to their attending physician and improved access to the healthcare system. In earlier qualitative studies, similar findings arose from students' perspectives, whereas our similar findings arose from patient perspectives. Earlier work in the US by Ogur and Hirsh described that students' longitudinal care transformed the student role and improved patients' experience of care [31]. Similarly, Hauer et al. reported that the LIC structure affords students opportunities to function in a doctor-like role and transmit and share information between the providers and patients [38].

The Western literature also reports patients' perspectives of having LIC students in their care. Flick et al. described the growth in student-patient relationships that develop over time and the symbiotic nature of the relationship; students' learning benefits from the longitudinal relationship and the LIC students' patients reported subjective improvement in health outcomes [33]. Poncelet et al. reported patients with more severity of illness described their longitudinal students as undertaking a physician-like role more than other patients who encountered students [32].

In the East Asian context of our study, Confucianism has profound influences on the patterns of government, society, education, and family. At the individual level, Confucian ethics is characterized by the promotion of virtue “De (德)" which consists of the five concepts: benevolence/humaneness "Ren (仁)", righteousness "Yi (義)”, proper rite “Li (禮)”, knowledge “Zhi (智)”, and integrity “Xin (信)". At a societal level, Confucianism emphasizes the importance of the family and social harmony, which is the practice of collectivism and regulated by loyalty “Zhong (忠)", filial piety “Xiao (孝)", contingency “Jie (節)", and righteousness “Yi (義)".

We consider our findings within our East Asian cultural context. Among the three themes we extracted from our patients' interviews, two themes connect closely to medical professionalism in Taiwan: companionship and empathy. We found that our patients valued students' considerable time keeping their company and understanding their feelings and emotions. This finding aligns with the study in the US, demonstrating that patients deeply valued the therapeutic alliances built with LIC students through longitudinal relationships [33]. Flick et al. describe that continuous and patientcentered care is the foundation of this alliance [33]. Does the "therapeutic alliance" in the Western context connect to our findings of "companionship and empathy"? We consider possible cultural underpinnings of the two latter themes derived inductively from our analysis. Following research from Ho et al. [19, 39] who describe the cultural influences on professionalism in Taiwan, we suggest that our Taiwanese patients' responses may be influenced by two sources: Confucian culture and perspectives on local health systems.

Confucianism underpins Taiwanese culture, the context where the interviewed patients received care $[40,41]$. The theme of companionship may relate to a Confucian focus that emphasizes the relationship between individuals and their society. In Taiwan, consistent with Confucian tradition, people recognize, expect, and seek individuals and their collective group to rely on each other mutually [42]. This "collectivism" contrasts with elements of Western individualism-particularly in the US where the earlier studies arose [23]. This difference may inform the meaning of our interviewee's responses. In an Asian context, the essence of the companionship expressed by the patients may 
relate to their appreciation when students "show deep presence" and connectivity, Confucian values that may be currently rare in the fast pace of Taiwanese medical practice. The interviewed patients from this cultural context described that the LIC student contributes to other forms of caring beyond the typical medical approach. We wonder if patients may sense that students filleven restore-a societal role through their longitudinal relationships.

The theme of empathy may relate to the Confucian notion of "Ren (仁)"-the central ethical principle of Confucian culture, "equivalent to the concepts of love, mercy, and humanity [43]." Ren is classically defined by a framing familiar to Western traditions: "Do not do to others what you do not want done to yourself" (己所不 欲勿施于人) [44]. In the Asian tradition, Ren relates directly to the concept of empathy as one of its core elements. In our study, patients specifically expressed valuing the Ren shown by the LIC students.

These patients' perceptions and expectations of the Taiwanese health system may also inform their responses. Currently, Taiwan is experiencing a severe physician shortage [45]; consequently, large medical institutions run at, and beyond, full capacity [46]. The care delivery system in Taiwan limits opportunities for longitudinal interpersonal relationships due to the pace of care. Outpatient clinics in tertiary hospitals are usually busy and crowded, and doctors may need to see 50 or more patients in a morning [47] (some specialists may see 30 patients in an hour [48]). Patients typically have very short visits with the doctor [48]. The patients' short contact time with physicians undermines patientphysician rapport; doctors have less time to act as companions or show empathy. Patients and doctors in Taiwan seek longer visits and long-term relationships, a circumstance not unlike the West. However, the meaning patients ascribe to deficits in physician companionship and empathy may be different in the Taiwanese context where the relationship is influenced by a Confucian sense of duty rather than a transaction.

\section{Limitations}

This study has limitations. Initially, students listed 57 longitudinally-tracked patients as eligible for this study, but only 20 patients (with their families, comprising 25 interviewees) agreed to be interviewed. We do not know the opinions of the patients who were not interviewed. It is possible that patients who did not agree to be interviewed may have different, even negative views, of longitudinal relationships with students. Our attending physicians determine which patients the LIC students follow longitudinally on the basis of the patients' suitability for interaction with medical students; this pool of patients may not represent the views of a general patient population in this hospital or elsewhere. Among those sampled, $72 \%$ were women, and we do not know the influence of sex on the results. We also do not know if the age distribution of our sample or other unmeasured demographic features represent the general patient population. We recognize that in 5 cases, patients and their family members were interviewed together, and in 4 cases, only family members were interviewed; we do not know the influence or effect of family opinions on the results. Considering reflexivity, our two qualitative researchers who performed the inductive analysis have connection to the LIC program. Although we used standard approach to analyze transcripts independently, we cannot exclude the possibility that our perceptions and consensus process contain biases. We note that this LIC progresses for 6 months, and LICs of greater length might have different impact on student-patient relationships. Finally, this study took place in one tertiary hospital in the largest city in Taiwan, and although it is the first such LIC patient study in Asia, we cannot assume the transferability of the results across other contexts.

\section{Conclusion}

Our study of patients' perspectives of LIC students suggests the value of care facilitation, companionship, and empathy in this Taiwanese care context. The LIC program creates a structure for medical students to establish longitudinal relationships with patients. It is our hope that longitudinal student-patient relationships extend the possibilities of the medical student role in Asia and beyond.

Abbreviations

LIC: Longitudinal integrated clerkship; TSGH: Tri-Service General Hospital

\section{Supplementary Information}

The online version contains supplementary material available at https://doi. org/10.1186/s12909-021-02553-7.

Additional file 1. Interview guide.

\section{Acknowledgements}

The authors would like to thank Professor Pauling Chu for his support and thank the study participants for their time.

\section{Authors' contributions}

Conception and design of study: YC, WT, SK; acquisition of data: YC, WF; analysis and interpretation of data: YC, WF, DAH, HL, WT, SK; drafting the manuscript: YC, DAH, HL, WT, SK; revision the manuscript: YC, DAH, HL, SK; approval of the version of the manuscript: YC, DAH, WF, HL, WT, SK. All authors have read and approved the manuscript.

\section{Funding}

This study was financially supported by grants from the Ministry of Science and Technology, Taiwan (MOST105-2511-S-016-002). The founder had no role in this study. 


\section{Availability of data and materials}

The datasets generated during and/or analysed during the current study available from the corresponding author on reasonable request.

\section{Declarations}

\section{Ethics approval and consent to participate}

This study was reviewed and approved by the Tri-Service General Hospital Institutional Review Board (TSGHIRB 2-105-05-050). Written informed consent was obtained from all participants.

\section{Consent for publication}

Not applicable.

\section{Competing interests}

Yaw-Wen Chang is director of TSGH's longitudinal integrated clerkship. David A. Hirsh is co-founder and director of Harvard Medical School's longitudinal integrated clerkship (the Cambridge Integrated Clerkship). Wen-Hui Fang is a preceptor the TSGH LIC. The authors alone are responsible for the content and writing of this article. All authors report no conflicts of interest and no financial disclosures.

\section{Author details}

'Department of Family and Community Medicine, Tri-Service General Hospital, National Defense Medical Center, No.325, Sec. 2, Chenggong Rd., Neihu Dist., Taipei City 11490, Taiwan, Republic of China. ${ }^{2}$ Graduate Institute of Medical Science, National Defense Medical Center, No.161, Sec. 6, Minquan E. Rd., Neihu Dist.,, Taipei City 11490, Taiwan, Republic of China. ${ }^{3}$ Harvard Medical School Academy, Tosteson Medical Education Center, Room 384, 260 Longwood Ave, Boston, MA 02115, USA. ${ }^{4}$ HMS-Cambridge Integrated Clerkship, Cambridge Health Alliance, 1493 Cambridge St, Cambridge, MA 02139, USA. Institute of International Healthcare Professionals Education and Research, China Medical University, No.77, Puhe Rd., Shenyang North New Area, Shenyang, Liaoning, P.R. China. ${ }^{6}$ School of Nursing, National Defense Medical Center, No.161, Sec. 6, Minquan E. Rd., Neihu Dist., Taipei City 11490, Taiwan, Republic of China. ${ }^{7}$ Graduate Institute of Life Science, National Defense Medical Center, No.161, Sec. 6, Minquan E. Rd., Neihu Dist., Taipei City 11490, Taiwan, Republic of China.

\section{Received: 11 May 2020 Accepted: 9 February 2021}

Published online: 10 March 2021

\section{References}

1. Hirsh D, Gaufberg E, Ogur B, Cohen P, Krupat E, Cox M, et al. Educational outcomes of the Harvard Medical School-Cambridge integrated clerkship: a way forward for medical education. Acad Med. 2012;87(5):643-50.

2. Hirsh D. Longitudinal integrated clerkships: Embracing the hidden curriculum, stemming ethical erosion, transforming medical education. In: Hafferty FW, O'Donnell JF, editors. The hidden curriculum in health professional education. edn. Hanover, NH: Dartmouth College Press; 2014. p. 193-202.

3. Gaufberg E, Bor D, Dinardo P, Krupat E, Pine E, Ogur B, et al. In pursuit of educational integrity: professional identity formation in the Harvard Medical School Cambridge integrated clerkship. Perspect Biol Med. 2017;60(2):258-74.

4. Hirsh DA, Walters LK. Learning in longitudinal integrated clerkships. In: Dent JA, Harden RM, Dan H, editors. A Practical Guide for Medical Teachers. 5th edn. Toronto: Elsevier; 2017. p. 84-91.

5. Alliance for Clinical Education. Longitudinal Integrated Clerkships: Principles, Outcomes, Practical Tools, and Future Directions, First edition. edn. North Syracuse: Gegensatz Press; 2016.

6. Hirsh DA, Ogur B, Thibault GE, Cox M. "Continuity" as an organizing principle for clinical education reform. N Engl J Med. 2007;356(8):858-66.

7. Ogur B, Hirsh D, Krupat E, Bor D. The Harvard Medical School-Cambridge integrated clerkship: an innovative model of clinical education. Acad Med. 2007;82(4):397-404.

8. Hirsh D, Walters L, Poncelet AN. Better learning, better doctors, better delivery system: possibilities from a case study of longitudinal integrated clerkships. Med Teach. 2012;34(7):548-54.

9. Chen HC, Teherani A. Workplace affordances to increase learner engagement in the clinical workplace. Med Educ. 2015;49(12):1184-6.
10. Latessa RA, Swendiman RA, Parlier AB, Galvin SL, Hirsh DA. Graduates' perceptions of learning affordances in longitudinal integrated clerkships: a dual-institution, Mixed-Methods Study. Acad Med. 2017;92(9):1313-9.

11. Walters L, Greenhill J, Richards J, Ward H, Campbell N, Ash J, et al. Outcomes of longitudinal integrated clinical placements for students, clinicians and society. Med Educ. 2012;46(11):1028-41.

12. Konkin J, Suddards C. Creating stories to live by: caring and professional identity formation in a longitudinal integrated clerkship. Adv Health Sci Educ Theory Pract. 2012;17(4):585-96.

13. O'Brien BC, Poncelet AN, Hansen L, Hirsh DA, Ogur B, Alexander EK, et al. Students' workplace learning in two clerkship models: a multi-site observational study. Med Educ. 2012;46(6):613-24.

14. Gaufberg E, Hirsh D, Krupat E, Ogur B, Pelletier S, Reiff D, et al. Into the future: patient-centredness endures in longitudinal integrated clerkship graduates. Med Educ. 2014;48(6):572-82.

15. Wamsley MA, Dubowitz N, Kohli P, Cooke M, O'Brien BC. Continuity in a longitudinal out-patient attachment for year 3 medical students. Med Educ. 2009;43(9):895-906.

16. Teherani A, Irby DM, Loeser H. Outcomes of different clerkship models: longitudinal integrated, hybrid, and block. Acad Med. 2013;88(1):35-43.

17. Norris TE, Schaad DC, DeWitt D, Ogur B, Hunt DD, Consortium of longitudinal integrated. Longitudinal integrated clerkships for medical students: an innovation adopted by medical schools in Australia, Canada, South Africa, and the United States. Acad Med. 2009;84(7):902-7.

18. Worley P, Couper I, Strasser R, Graves L, Cummings BA, Woodman R, et al. A typology of longitudinal integrated clerkships. Med Educ. 2016;50(9):922-32.

19. Ho MJ, Yu KH, Hirsh D, Huang TS, Yang PC. Does one size fit all? Building a framework for medical professionalism. Acad Med. 2011;86(11):1407-14.

20. Leung DC, Hsu EK, Hui EC. Perceptions of professional attributes in medicine: a qualitative study in Hong Kong. Hong Kong Med J. 2012;18(4): 318-24.

21. Jha V, McLean M, Gibbs TJ, Sandars J. Medical professionalism across cultures: a challenge for medicine and medical education. Med Teach. 2015; 37(1):74-80.

22. Al-Rumayyan A, Van Mook W, Magzoub ME, Al-Eraky MM, Ferwana M, Khan MA, et al. Medical professionalism frameworks across non-Western cultures: A narrative overview. Med Teach. 2017;39(sup1):S8-S14.

23. Nilchaikovit T, Hill JM, Holland JC. The effects of culture on illness behavior and medical care. Asian and American differences. Gen Hosp Psychiatry. 1993;15(1):41-50.

24. Pun JKH, Chan EA, Wang S, Slade D. Health professional-patient communication practices in East Asia: an integrative review of an emerging field of research and practice in Hong Kong, South Korea, Japan, Taiwan, and mainland China. Patient Educ Couns. 2018;101(7): 1193-206.

25. Ho MJ, Lin CW, Chiu YT, Lingard L, Ginsburg S. A cross-cultural study of students' approaches to professional dilemmas: sticks or ripples. Med Educ. 2012;46(3):245-56.

26. Monrouxe LV, Chandratilake M, Gosselin K, Rees CE, Ho MJ. Taiwanese and Sri Lankan students' dimensions and discourses of professionalism. Med Educ. 2017;51(7):718-31.

27. Pan H, Norris JL, Liang YS, Li JN, Ho MJ. Building a professionalism framework for healthcare providers in China: a nominal group technique study. Med Teach. 2013;35(10):e1531-6.

28. Hu Y, Zhang Z. Patient education - a route to improved patient experience in Chinese hospitals? Patient Educ Couns. 2015;98(12):1651-2.

29. Luxford K, Safran DG, Delbanco T. Promoting patient-centered care: a qualitative study of facilitators and barriers in healthcare organizations with a reputation for improving the patient experience. Int J Qual Health Care. 2011;23(5):510-5.

30. Walters L, Hirsh D. Teaching in general practice: considering conceptual lenses. Med Educ. 2011;45(7):660-2.

31. Ogur B, Hirsh D. Learning through longitudinal patient care-narratives from the Harvard Medical School-Cambridge integrated clerkship. Acad Med. 2009;84(7):844-50.

32. Poncelet AN, Wamsley M, Hauer KE, Lai C, Becker T, O'Brien B. Patient views of continuity relationships with medical students. Med Teach. 2013;35(6): 465-71.

33. Flick RJ, Felder-Heim C, Gong J, Corral J, Kalata K, Marin A, et al. Alliance, trust, and loss: experiences of patients cared for by students in a longitudinal integrated clerkship. Acad Med. 2019;94(11):1806-13. 
34. Hudson JN, Knight PJ, Weston KM. Patient perceptions of innovative longitudinal integrated clerkships based in regional, rural and remote primary care: a qualitative study. BMC Fam Pract. 2012;13:72.

35. Taipei City Government. Demographic Overview. 2020. [https:/english.gov. taipei/cp.aspx?n=C619997124A6D293]. Accessed 30 Apr 2020.

36. Miles MB, Huberman AM. Qualitative data analysis : an expanded sourcebook. 2nd ed. Thousand Oaks: Sage Publications; 1994.

37. Thomas DR. A general inductive approach for analyzing qualitative evaluation data. Am J Eval. 2006;27(2):237-46.

38. Hauer KE, Hirsh D, Ma I, Hansen L, Ogur B, Poncelet AN, et al. The role of role: learning in longitudinal integrated and traditional block clerkships. Med Educ. 2012;46(7):698-710.

39. Ho MJ, Yu KH, Pan H, Norris JL, Liang YS, Li JN, et al. A tale of two cities: understanding the differences in medical professionalism between two Chinese cultural contexts. Acad Med. 2014;89(6):944-50

40. Chen C-Y. Development of Confucianism in Taiwan. Contemp Chin Thought. 2009:41(1):10-27.

41. Huang C-C. The Confucian tradition and prospects for Taiwan in the twenty-first century. Contemp Chin Thought. 2009:41(1):70-90.

42. CMADS P, Bernardes FM, Minari AG, Silva CHM, Paro HBM. Innovations in curriculum designs do not guarantee students' patient-centered attitudes running title: curricula and patient-centered attitudes. Revista Brasileira De Educ Médica. 2019;43(4):167-75.

43. Cultural Beliefs and Practices: Role of Confucianism. Stanford School of Medicine Ethnogeriatrics Web site. [https://geriatrics.stanford.edu/ ethnomed/chinese/fund/beliefs.html]. Accessed 30 Apr 2020.

44. Gardner DK, Confucius. Zhu Xi's Reading of the analects : canon, commentary, and the classical tradition. New York: Columbia University Press; 2003.

45. Cheng T-M. Taiwan's health care system: the next 20 years. Brookings Web site. 2015; [https://www.brookings.edu/opinions/taiwans-health-care-systemthe-next-20-years/]. Accessed 30 Apr 2020.

46. Jan CF, Chiu TY, Chen CY, Guo FR, Lee MC. A 10-year review of health care reform on family practice integrated care project-Taiwan experience. Fam Pract. 2018;35(4):352-7.

47. Wu TY, Majeed A, Kuo KN. An overview of the healthcare system in Taiwan. London J Prim Care (Abingdon). 2010:3(2):115-9.

48. American Institue in Taiwan. Medical assistance. American Institue in Taiwan Web site. [https://www.ait.org.tw/u-s-citizen-services/doctors/]. Accessed 30 Apr 2020.

\section{Publisher's Note}

Springer Nature remains neutral with regard to jurisdictional claims in published maps and institutional affiliations.

Ready to submit your research? Choose BMC and benefit from:

- fast, convenient online submission

- thorough peer review by experienced researchers in your field

- rapid publication on acceptance

- support for research data, including large and complex data types

- gold Open Access which fosters wider collaboration and increased citations

- maximum visibility for your research: over $100 \mathrm{M}$ website views per year

At $\mathrm{BMC}$, research is always in progress.

Learn more biomedcentral.com/submissions 